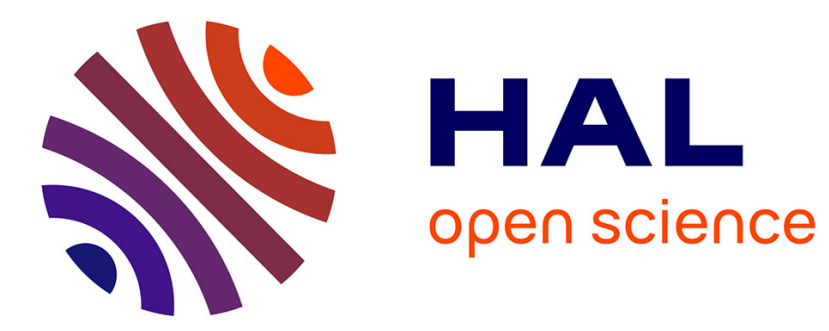

\title{
Shared Risk Resource Groups and Survivability in Multilayer Networks
}

David Coudert, Stéphane Pérennes, Hervé Rivano, Marie-Emilie Voge

\section{To cite this version:}

David Coudert, Stéphane Pérennes, Hervé Rivano, Marie-Emilie Voge. Shared Risk Resource Groups and Survivability in Multilayer Networks. International Conference on Transparent Optical Networks (ICTON), Jun 2006, Nottingham, United Kingdom. pp.235 - 238, 10.1109/ICTON.2006.248442 . inria-00429170

\section{HAL Id: inria-00429170 \\ https://hal.inria.fr/inria-00429170}

Submitted on 1 Nov 2009

HAL is a multi-disciplinary open access archive for the deposit and dissemination of scientific research documents, whether they are published or not. The documents may come from teaching and research institutions in France or abroad, or from public or private research centers.
L'archive ouverte pluridisciplinaire HAL, est destinée au dépôt et à la diffusion de documents scientifiques de niveau recherche, publiés ou non, émanant des établissements d'enseignement et de recherche français ou étrangers, des laboratoires publics ou privés. 


\title{
Shared Risk Resource Groups and Survivability in Multilayer Networks
}

\author{
David Coudert, Stéphane Pérennes, Hervé Rivano, Marie-Emilie Voge \\ Mascotte project, CNRS/INRIA/UNSA, \\ 2004 route des Lucioles, B.P. 93, 06902 Sophia Antipolis Cedex, France. \\ E-mail: Firstname.Lastname@sophia.inria.fr
}

\begin{abstract}
Failure resilience is a desired feature of the Internet. Most traditional restoration architectures are designed assuming single failure cases, which is not adequate in present day multilayer networks. Multiple link failure models, such as Shared Risk Link Groups (SRLG), Shared Risk Node Groups (SRNG), and more generally Shared Risk Resource Groups (SRRG), are becoming critical in survivable network design. These shared risk models have been unified through the notion of colored graphs. In this paper we provide an efficient MILP formulation for the minimum color path problem which is related to finding a path of maximum reliability in a multilayer network and is thus essential issue of network survivability.
\end{abstract}

\section{INTRODUCTION}

Modern day networks are multilayer networks where traffic flows are routed on meshed topologies whose links are indeed end-to-end paths on a high bandwidth infrastructure. IP/WDM, MPLS or MP $\lambda$ S networks, as well as P2P or GRID computing overlay structures are example of such a hierarchy.

In these settings, customers expect an uninterrupted service, even in the event of failures such as power outages, equipment failures, natural disasters and cable cuts. Many layer wide protection schemes have been proposed in the literature, as for instance for the WDM optical networks [1].

One method to provide survivability is through path protection schemes, in which a disjoint backup path is precomputed for every working path. Such protection schemes provide $100 \%$ reliability against any single link failure in the network layer that is considered. However, in multilayer network settings, a single failure event in the underlying layer may result in the failure of several links in the virtual network. For example, in a MPLS/WDM network, several apparently independent label switched paths (LSP) may be routed on the same fiber. Even though these LSPs represent disjoint links of the MPLS virtual topology, the cut of an optical fiber can cause all the LSPs to fail. Such a group of links sharing a common risk factor is said to be a Shared Risk Link Group [2]. When the risk of failure concerns nodes instead of links the group is said to be a Shared Risk Node Group, and more generally the notion of Shared Risk Resource Group (SRRG) has been defined to encompass any kind of resources sharing a common risk of unavailability. Recently in $[3,4]$ the SRRG notion has been formalized through colored graphs.

Indeed, colored graphs model any application in which the links of a network belong to one or more SRRG. Each SRRG is associated to a color and each link of the network is assigned colors according to which SRRG it belongs. Thanks to simple graph transformations the network can then be modeled by a colored graph whose links are monochromatic (see $[3,4]$ for more details).

Many problems have been studied to answer survivability challenges in the SRRG context, such as the diverse routing problem also called the color disjoint paths problem. It consists in finding two paths between a pair of nodes in the virtual network such that no single failure in the underlying layer may cause both paths to fail simultaneously. This problem is much more difficult than the traditional disjoint path problem of graph theory [4,5] and recent studies have proven its NP-completeness [6,7]. In [4], it was shown that it is NP-hard to approximate the maximum number of disjoint paths in the SRRG settings: that is no polynomial time algorithm can provide solutions which are guaranteed to be lower than the optimal number of paths multiplied by some factor depending on the size of the graph.

This fosters the relevance of the different heuristic approaches studied for this problem $[3,5]$. One of the common problems that arise in restoration path computation is the existence of a trap topology [8]: if a service path is routed over a trap topology, then there may not exist a diverse restoration path, even though two diverse paths exist in the network.

A challenge that SRRG protection schemes have to face consists in the impossibility to provide $100 \%$ reliability against certain multiple link failure events, depending on the SRRG configuration [3]. In these cases, an objective may be to find one or more paths for each connection, such that the reliability for each connection is maximized: it is the minimum overlapping paths problem. It consists in finding a set of paths sharing a minimum

The research reported in this paper is funded in part by ACI-SI PRESTO, Région PACA, France Telecom R\&D, European projects IST FET AEOLUS and COST 293 Graal. 
number of SRRG, or colors. This problem as well as the minimum cost SRRG diverse routing problem and the routing problem under both link capacity and path length constraints have also been shown to be NP-complete [6].

In this work, we are concerned with maximizing the reliability of connections against failure events in which multiple links may fail simultaneously. We therefore focus on the minimum color path problem and present efficient MILP formulations for practical cases.

\section{COMBINATORIAL MODEL AND ALGORITHMIC COMPLEXITY}

Shared Risk Resources Groups are naturally modeled by associating to each group, or risk, a color, and to each edge, or resource, the colors representing the risks affecting it $[3,4]$. According to the general case of Shared Risk Resource Groups, an edge may belong to several colors, modeling the fact that a resource may face different and independent risks. However, using straightforward graph transformations, one can assume without loss of generality that edges are monochromatic. In other words, the colors partition the edge set.

A colored graph is henceforth a triplet $\mathrm{G}=(\mathrm{V}, \mathrm{E}, \mathrm{C})$ where $(\mathrm{V}, \mathrm{E})$ is an undirected graph and $C$ is a partition of $\mathrm{E}$. A weighted colored graph is a colored graph such that each color $c \in C$ has a weight $w(c) \geq 0$. The span of a color is the number of connected components of the subgraph induced by the edges of this color.

Using this combinatorial framework, several network survivability issues have been studied. Table 1 summarizes the results that are available in the literature and in [4]. These problems are well-known in classical graph theory, i.e. when considering single failure cases. One can immediately note that the complexity and approximability properties of these problems change dramatically. The core difficulty of these problems can be explained by the fact that the structure of the colors overcomes the topology of the graph. More precisely, this implies a strong proximity to Min Set Cover (MSC) problem [4]. Indeed, most of these problems can be reduced to MSC, and the Min Color Spanning Tree problem is exactly equivalent to MSC.

Consequently, the structure of the colors has a strong influence on the complexity: most of the easy cases where polynomial exact or approximated algorithms exist are induced by colors of span 1 or bounded by a given constant $k$. As a matter of fact, in the case of colors of span 1 , since each color is a single connected component, the topology of the graph can be exploited again.

\begin{tabular}{|c|c|c|c|c|c|c|}
\hline \multicolumn{2}{|c|}{ Min Color... } & Path & 2-Disjoint Paths & st-Cut & Cut & Spanning Tree \\
\hline \multirow{3}{*}{ General } & Complexity & NP-Hard [3] & NP-Hard [3] & NP-Hard & $?$ & NP-Hard \\
\hline & Inapprox. & $2^{\log ^{1-\delta}(n)}$ & $?$ & $2^{\log ^{1-\delta}(n)}$ & $?$ & $\mathrm{O}(\log \mathrm{n})$ \\
\hline & Approx. & $?$ & $?$ & $?$ & $?$ & $\mathrm{O}(\log \mathrm{n})$ \\
\hline \multirow{3}{*}{ Span k } & Complexity & NP-Hard & $?$ & NP-Hard & $?$ & NP-Hard \\
\hline & Inapprox. & $\exists \varepsilon>0, k^{\varepsilon}$ & $?$ & $\exists \varepsilon>0, k^{\varepsilon}$ & $?$ & $\mathrm{O}(\log \mathrm{n})$ \\
\hline & Approx. & $\mathrm{k}$ & $?$ & $\mathrm{k}$ & $?$ & $\mathrm{O}(\log \mathrm{n})$ \\
\hline \multirow{3}{*}{ Span 1} & Complexity & \multirow{3}{*}{$\mathrm{P}[9]$} & \multirow{3}{*}{$\mathrm{P}$} & \multirow{3}{*}{$\mathrm{P}$} & \multirow{3}{*}{$\mathrm{P}$} & NP-Hard \\
\hline & Inapprox. & & & & & $\mathrm{O}(\log \mathrm{n})$ \\
\hline & Approx. & & & & & $\mathrm{O}(\log \mathrm{n})$ \\
\hline \multicolumn{2}{|c|}{ Bounded degree } & -- & -- & $P$ & $P$ & -- \\
\hline
\end{tabular}

Table 1: Complexity and approximability properties of colored problems. 


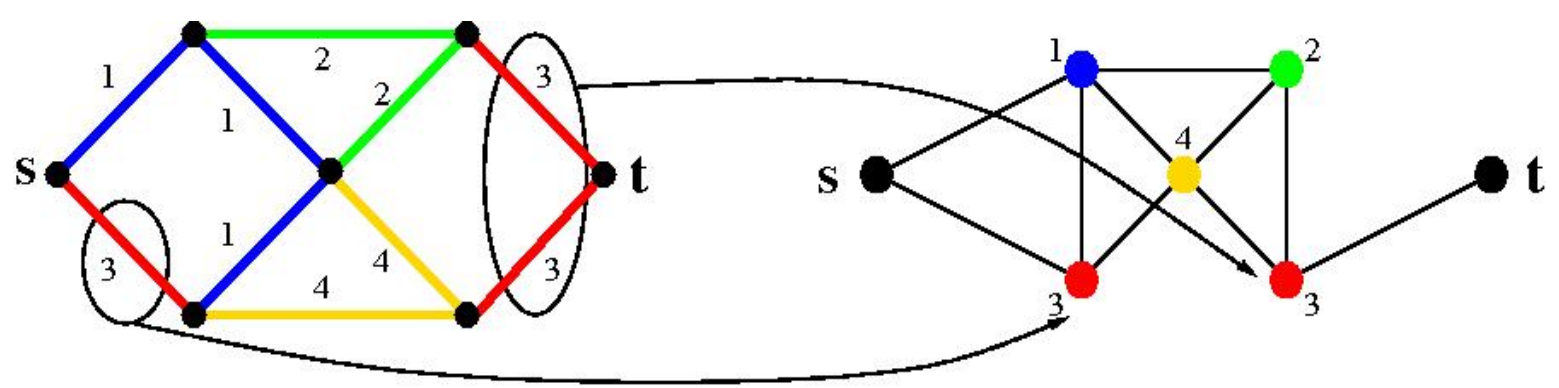

Fig 1: A colored graph and the collapsed graph of the color components.

\section{COPING WITH MINIMUM COLOR PATH}

According to Table 1, the Min Color Path problem is NP-complete in the general case. Consequently several heuristic algorithms have been proposed in the literature $[1,3,4,7,9]$. Indeed, despite the theoretical complexity of the problem, algorithms computing good color paths are still needed.

In this section, we focus on MILP formulations of the problem where the number of binary variables is kept as low as possible, so that optimal or near optimal solutions can be computed efficiently.

\section{General MILP formulation}

In the general case, one can write the Min Color Path problem as the following flow linear problem with binary variables allowing for keeping track of the colors that are used. This formulation uses $|C|$ binary variables that cannot be relaxed. On the other hand, the flow variables can be fractional since the solution is a single path.

\begin{tabular}{|l|l|}
\hline Flow variables & $\forall(u, v) \in E, f(u, v)=-f(v, u)$ \\
\hline Capacity constraints & $-1 \leq f(u, v) \leq 1$ \\
\hline Flow conservation & $\forall u, \sum f(u, v)=0, \quad 1 \quad$ if $\quad s, \quad-1 \quad$ if $t$ \\
\hline Binary variables & $\forall c \in C, \quad \forall(u, v) \in c, \quad|f(u, v)| \leq X_{c}$ \\
\hline Objective & Minimize $\sum X_{c}$ \\
\hline
\end{tabular}

\section{Reducing the number of binary variables}

The computation time for solving such mathematical optimization problem strongly depends on the number of binary variables it contains. In the previous MILP, the binary variables are used at two distinct level. On the one hand, they correlate the different connected components of a color. On the other hand, they correlate the edges of each connected component. The component-wise level is at the heart of colored graphs while the edge-wise level can be taken into account with classical graph algorithms. Indeed, the core of Min Color Path problem is to find the minimum set of colors which contains a path.

In the following, we present a sophisticated approach combining a MILP optimization and algorithmic graph transformations allowing for a significant gain on efficiency. Indeed, we decompose the problem in two stages. First, an optimization process focuses on finding the minimum set of colors containing a s-t path. The actual path is computed afterward with a classical shortest path algorithm.

In order to get an efficient MILP formulation with as few binary variables as possible, a transformation of the graph is done as illustrated in Figure 1.

The main idea consists in collapsing each component of a color into a single vertex connected to the vertices related to adjacent component of colors. In this graph, a color of span 1 is collapsed into a single vertex (e.g. color 1), while a color of span $k$ is transformed into $k$ separate vertices (e.g. color 3 ). If all colors had span 1 , the minimum set of colors containing a s-t path would be collapsed into a shortest path. This confirms that the MCP problem is polynomial in this case. In particular, the problem can still be written as a flow problem, but there is no need for binary variables since each color is a single vertex. When some colors have span more than 1 , binary variables are needed again, to correlate the vertices related to the different connected components of a color. 
Consequently, the following MILP finds the minimum number of colors containing a s-t path with only a binary variable per color of span more than 1 . As an illustration, only one binary variable is required for solving the example of Figure 1, against 4 with the first formulation. This formulation is hence solved efficiently when the number of such colors is low. This fortunately seems to be the case in practical instances $[1,7]$.

This also proves that the Minimum Color Path problem is polynomial when the number of colors of span more than 1 is bounded by a given constant $k$.

\begin{tabular}{|l|l|}
\hline Flow variables & $\forall(u, v) \in E, f(u, v)=-f(v, u)$ \\
\hline Capacity constraints & $-1 \leq f(u, v) \leq 1$ \\
\hline Flow conservation & $\forall u, \sum f(u, v)=0, \quad 1 \quad$ if $\quad s, \quad-1 \quad$ if $t$ \\
\hline Color variables & $\forall c \in C, \quad \forall v \in \Gamma\left(u_{c}\right), \quad\left|f\left(u_{c}, v\right)\right| \leq X_{c}$ \\
\cline { 2 - 2 } & $X_{c} \in[0,1]$ if span $1, \quad\{0,1\} \quad$ otherwise \\
\hline Objective & Minimize $\sum X_{c}$ \\
\hline
\end{tabular}

\section{CONCLUSION}

In this paper, we have investigated combinatorial problems yielded by network survivability issues in multilayer networks facing Shared Risk Resource Group failures. This work has been realized in the "colored graphs" theoretical framework. We have proposed a sophisticated optimization process for the Minimum Color Path problem which combines an algorithmic graph transformation and an efficient MILP formulation taking into account the span of the colors. This formulation uses a reduced number of binary variables, which proves that instances with a bounded number of colors of span larger that 1 can be solved in polynomial time.

Network survivability issues give rise to many other combinatorial problems that can be modeled through SRRG and colored graphs. Most of these problems have been proved NP-hard and hard to approximate in the literature. Therefore, finding efficient algorithms for computing optimal or near optimal solutions is a challenging line of research. Conducting thorough analysis of the underlying optimization problems may allow for isolating the heart of the difficulties and designing efficient MILP formulation. In particular, the approach that has been followed in this paper should give promising results on the Color Disjoint Path and Minimum Overlapping Paths problems which are fundamental issues for network survivability.

\section{REFERENCES}

[1] A. Todimala and B. Ramamurthy: Survivable Virtual Topology Routing under Shared Risk Link Groups in WDM Networks. In Proc. BroadNets, pages 103-139, 2004.

[2] D. Papadimitriou, et al.: Inference of Shared Risk Link Groups. IETF Draft, OIF 2001-066, 2001.

[3] S. Yuan, S. Varma, and J.P. Jue: Minimum-Color Path Problems for Reliability in Mesh Networks. In Proc. IEEE InfoCom, 2005.

[4] D. Coudert, et al.: Complexity and approximability issues of Shared Risk Resource Group. Technical report, INRIA Research Report 5859 and I3S Research Report I3S/RR-2003-08-FR, March 2006.

[5] R. Bhandari: Survivable Networks: Algorithms for Diverse Routing. Kluwer Academic, 1999.

[6] J.Q. Hu: Diverse routing in mesh optical networks. IEEE/ACM Transaction on Communications, 51(3):489-494, 2003.

[7] L. Shen, X. Yang and B. Ramamurthy: Shared Risk Link Group (SRLG)-Diverse Path Provisioning under Hybrid Service Level Agreements in Wavelength-Routed Optical Mesh Networks. IEEE/ACM Transaction on Networking. (to appear).

[8] D. Xu, Y. Xiong and C. Qiao: Protection with multi-segments (PROMISE) in Networks with Shared Risk Link Groups (SRLG). IEEE/ACM Transaction on Networking, 11(2):248-258, 2003.

[9] P. Datta and A.K. Somani: Diverse Routing for Shared Risk Resource Groups (SRRG) failures in WDM Optical Networks. In Proc. BroadNets, 2004. 